\title{
Low-spin state stabilization in a mononuclear iron(II) complex and high- temperature cooperative spin crossover mediated by hydrogen bonding
}

\author{
Sipeng Zheng, ${ }^{a}$ Niels R. M. Reintjens, ${ }^{a}$ Maxime A. Siegler, ${ }^{b}$ Olivier Roubeau, ${ }^{c}$ Elisabeth Bouwman, ${ }^{a}$ \\ Andrii Rudavskyi, ${ }^{d}$ Remco W. A. Havenith, ${ }^{d, e}$ and Sylvestre Bonnet ${ }^{a} *$ \\ ${ }^{a}$ Leiden Institute of Chemistry, Gorlaeus Laboratories, Leiden University, P.O. Box 9502, Leiden, \\ 2300 RA, The Netherlands \\ ${ }^{\mathrm{b}}$ Small Molecule X-ray Facility, Department of Chemistry, Johns Hopkins University, Baltimore, \\ MD 21218, USA \\ ${ }^{\mathrm{c}}$ Departamento de Física de la Materia Condensada and Instituto de Ciencia de Materiales de \\ Aragón, Universidad de Zaragoza, Pedro Cerbuna 12, E-50009 Zaragoza, Spain \\ ${ }^{d}$ Theoretical Chemistry, Zernike Institute for Advanced Materials, University of Groningen, \\ Nijenborgh 4, 9747 AG Groningen, The Netherlands \\ ${ }^{\mathrm{e}}$ Stratingh Institute for Chemistry, University of Groningen, Nijenborgh 4, 9747 AG Groningen, \\ The Netherlands and Ghent Quantum Chemistry Group, Department of Inorganic and Physical \\ Chemistry, Ghent University, Krijgslaan 281 (S3), B-9000 Gent, Belgium \\ Email: bonnet@chem.leidenuniv.nl \\ Fax: +31715274451 Tel: +31715274260
}

\begin{abstract}
In this work the new tetrapyridyl ligand bbpya and its mononuclear coordination compound [Fe(bbpya)(NCS) $)_{2}$ (1) were prepared. According to magnetic susceptibility, differential scanning calorimetry fitted to Sorai's domain model, and powder X-ray diffraction measurements, 1 is lowspin at room temperature and exhibits a spin crossover transition at an exceptionally high transition temperature of $T_{1 / 2}=418 \mathrm{~K}$. Although the SCO of 1 spans over a temperature range of more than $150 \mathrm{~K}$, it is characterized by a wide $(21 \mathrm{~K})$ and dissymmetric hysteresis cycle, thus showing cooperativity. The crystal structure of the LS phase of 1 shows strong N-H...S intermolecular Hbonding interactions that explain, at least in part, the cooperative SCO behaviour observed for $\mathbf{1}$. DFT and CASPT2 theoretical calculations in vacuum demonstrate that bbpya generates a stronger ligand field around iron(II) compared to its analogue bapbpy, which stabilizes the LS state and destabilizes the HS state in 1 compared to [Fe(bapbpy)(NCS) $)_{2}$ (2). Secondly, periodic DFT calculations suggest that crystal-packing effects are significant for compound $\mathbf{2}$ where they destabilize the HS state by about $1500 \mathrm{~cm}^{-1}$. Such crystal packing effects may explain the much lower transition temperature found for the SCO of 2.
\end{abstract}




\section{Introduction}

Spin crossover ( $\mathrm{SCO}$ ) consists in the switching of $3 \mathrm{~d}^{4}-3 \mathrm{~d}^{7}$ transition metal ions between a high-spin state (HS) and a low-spin state (LS) ${ }^{[1]}$. It can be triggered by temperature variations, by light irradiation, or by the application of pressure, of a magnetic field, or of an electric field. ${ }^{[2]}$ In cooperative SCO materials the switching of a particular metal ion is influenced by the spin state of its neighbors, ${ }^{[3]}$ which may lead, at the macroscopic level, to abrupt transitions and hysteresis. Cooperativity is a very important aspect of SCO, as it is required in many technological applications involving SCO for which bistability is necessary, such as displays or memory devices. ${ }^{[4]}$ However, it is difficult to design cooperative SCO materials with transitions occurring at or above room temperature. ${ }^{[5]}$ Coordination polymers such as for example iron(II) 1,2,4-triazole ${ }^{[6]}$ and Hofmann networks of the type $\left[\mathrm{Fe}^{\mathrm{II}}\left(\mu-N, N^{\prime} \text {-bis-diimine }\right) \mathrm{M}(\mu-\mathrm{CN})_{4}\right]^{[7]}(\mathrm{M}=\mathrm{Cu}, \mathrm{Ni}, \mathrm{Pt}, \mathrm{Au}, \mathrm{Ag})$ are classical examples of cooperative SCO compounds in which the bridging ligands allow for efficient communication between iron centers. However, in such coordination polymers the ions situated at the boundaries of a crystalline domain may feel a different environment compared to the ions in the bulk, which can be detrimental to SCO nanomaterials. ${ }^{[8]}$ Mononuclear compounds, on the contrary, offer a well-defined coordination environment throughout the whole solid-state material. However, molecular compounds with cooperative transitions occurring near or above room temperature remain scarce..$^{[5,9-15]}$ On the one hand the ligand set must stabilize the LS state of iron(II); on the other hand it should allow very strong supramolecular interaction to counter-balance the natural narrowing of hysteresis cycles when the transition temperature of the SCO increases. ${ }^{[16]}$ A seminal SCO paper ${ }^{[4]}$ even stated that "if [SCO] molecules are hydrogen-bonded, [intermolecular] interactions may be enhanced, but remain insufficient to give rise to a strong cooperativity." In this paper, we describe [Fe(bbpya)(NCS) 2 (1), a new molecular SCO compound that shows at the same time an exceptionally high transition temperature, high cooperativity, and intermolecular H-bonding, and we demonstrate with theoretical calculations that the bbpya stabilizes the LS state of the iron(II) complex.

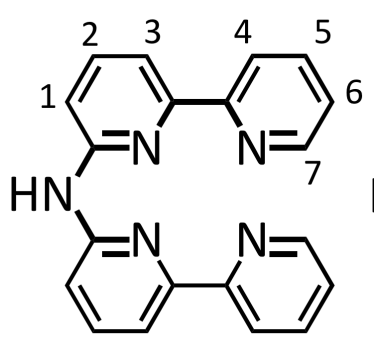

bbpya

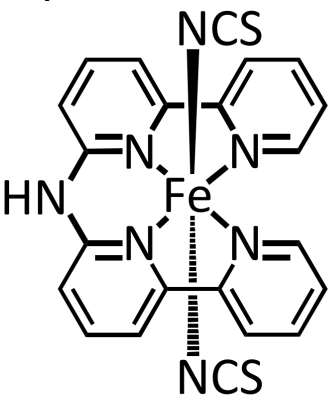

$\left[\mathrm{Fe}\left(\right.\right.$ bbpya) $\left.(\mathrm{NCS})_{2}\right]$ 1

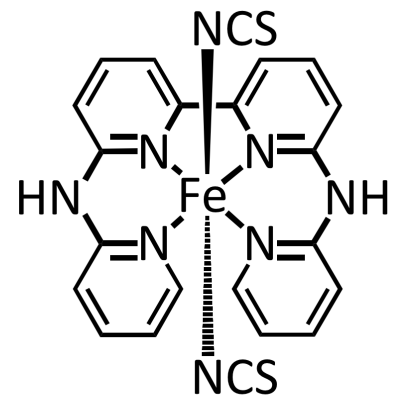

$\left[\mathrm{Fe}\left(\right.\right.$ bapbpy)$\left.(\mathrm{NCS})_{2}\right]$

Scheme 1. Schematic drawing of the bbpya ligand, of its iron complex 1, and of the analogue compound [Fe(bapbpy)(NCS $\left.)_{2}\right](2) .{ }^{[17]}$ Proton numbering scheme is shown for NMR attributions.

Inspired by earlier investigations on the two-step SCO compound [Fe(bapbpy)(NCS) 2 (2, see Scheme 1$)^{[17]}$ the new rigid $\mathrm{N}_{4}$-donor ligand bbpya was designed, namely $N, N$-bis(2,2'-bipyrid-6yl)amine (Scheme 1). This tetradentate ligand consists of two bipyridines connected by an $\mathrm{N}-\mathrm{H}$ bridge aimed at forming intermolecular $\mathrm{N}-\mathrm{H} \cdots \mathrm{S}$ hydrogen bond interactions, which have been shown to be critical for the cooperativity of bapbpy-based SCO compounds. ${ }^{[18]}$ Bbpya is rather similar to bapbpy and is expected to coordinate to iron(II) in a tetradentate fashion, leaving two trans axial positions for the binding of thiocyanate ions. However, bbpya also differs from bapbpy: it has only one $\mathrm{N}-\mathrm{H}$ bridge and forms upon coordination two 5-membered and one 6-membered rings, whereas bapbpy has two $\mathrm{N}-\mathrm{H}$ bridges and forms one 5-membered and two 6-membered rings upon coordination (Scheme 1). The presence of two bpy chelates, and the overall more open structure of bbpya, are expected to result in a reduced distortion of the coordination sphere of iron(II), an increase of the ligand field splitting, and therefore a stabilization of the LS state. 


\section{Results and discussion}

The ligand bbpya was obtained in $69 \%$ yield by a Buchwald-Hartwig cross-coupling reaction between 6-amino-2,2'-bipyridine ${ }^{[19,20]}$ and 6-bromo-2,2'-bipyridine (Figure 1). The reaction of bbpya with 1.1 eq. of $\mathrm{Fe}(\mathrm{NCS})_{2}$ in $\mathrm{MeOH}$ resulted in a deep purple suspension, which was stirred overnight and filtered to afford compound $\mathbf{1}$ as a deep purple powder. The dark color of the compound suggested a low-spin state at room temperature. Single crystals suitable for X-ray structure determination were grown using liquid-liquid diffusion of a methanolic solution of $\left[\mathrm{Fe}(\mathrm{NCS})_{2}\right]$ into a DMF solution of bbpya. The powder X-ray diffraction pattern calculated from the single crystal data determined at 110(2) K matches the experimental powder X-ray diffractogram of compound 1 at $298 \mathrm{~K}$. Furthermore, identical IR spectra were obtained for the crude powder and for the single crystals (Figure S1 and S2). Altogether, at room temperature and below both the crystals and the powder sample of $\mathbf{1}$ were made of the same phase of the same compound.

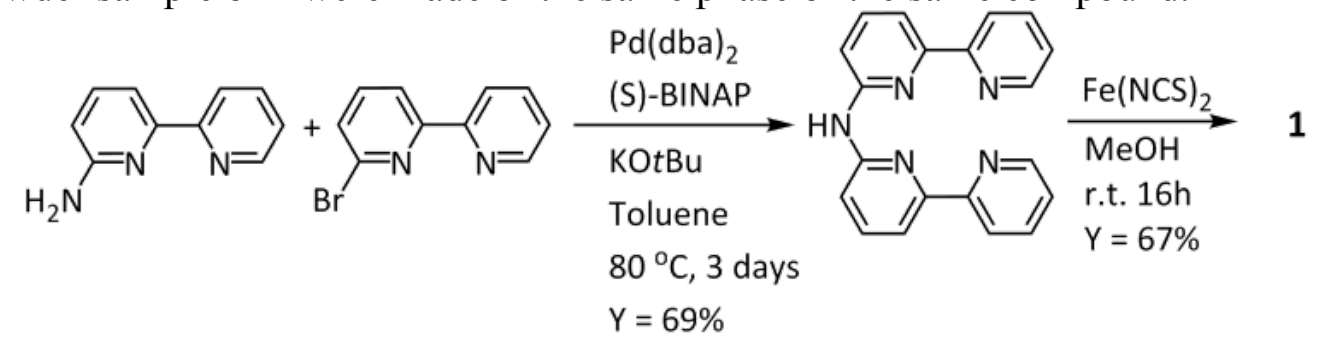

Figure 1. Synthetic route towards the ligand bbpya and its iron complex $\mathbf{1}$.

Compound 1 crystallizes in the triclinic space group $P-1$. In the structure of 1 at $110(2) \mathrm{K}$, the complex is disordered, and both components of the disorder are related via a pseudo-inversion center [occupancy factor of the major component: $0.7038(15)$ ]. The coordination geometry and bond distances in the two orientations are highly similar, but the two orientations have a major impact on the long-range hydrogen bonding interactions (see below). As anticipated, the Fe(II) ion is described in an octahedral geometry with four N-donors of the ligand bbpya in the equatorial plane, and two N-donors of the thiocyanate anions in the axial positions. The crystal lattice does not contain any solvent molecules. A projection of complex $\mathbf{1}$ is shown in Figure 2a (see Table 1 for bond lengths and angles). At $110 \mathrm{~K}$ the average $\mathrm{Fe}-\mathrm{N}$ bond length is $1.96 \AA$, which is typical for a LS Fe(II) complex in an octahedral environment, and comparable to those found for 2 in the LS phase $\left(\mathrm{Fe}-\mathrm{N}_{\mathrm{avg}}=1.95 \AA\right) .{ }^{[17]}$ The angle between the two planes of the two terminal pyridine rings is $22.8^{\circ}$ in $\mathbf{1}$, which is significantly smaller than that found in $2\left(44.8^{\circ}\right) .^{[17]}$ The more planar orientation of the coordinated bbpya ligand is likely due to the replacement of one of the sixmembered ring in $\mathbf{2}$ by a five-membered ring in $\mathbf{1}$ (see above). Unlike in $\mathbf{2}$, in $\mathbf{1}$ the angles between the vectors Fe1-N6 or Fe1-N7 and the mean molecular plane defined by N1, N2, N4 and N5, are 82.5 or $84.2^{\circ}$, respectively, i.e., the axial thiocyanate ligands are approximately perpendicular to the mean molecular plane of bbpya. Side views of the molecular geometries of $\mathbf{1}$ and $\mathbf{2}$ at $110 \mathrm{~K}$ (Figure $2 \mathrm{~b})$ show that $\mathbf{1}$ has indeed a much less distorted octahedron than $\mathbf{2}$.

Table 1. Selected bond lengths $(\AA)$ and angles $\left(^{\circ}\right)$ for the crystal structure of complex 1 at $110(2) \mathrm{K}$. Short intermolecular interactions $(\AA)$ of complex 1 are also provided.

\begin{tabular}{lccccc}
\hline Fe1-N1 & $1.996(4)$ & $\mathrm{N} 3 \ldots \mathrm{S} 2$ & $3.321(4)$ & $\mathrm{N} 6-\mathrm{Fe} 1-\mathrm{N} 7$ & $178.48(12)$ \\
Fe1-N2 & $1.934(4)$ & $\mathrm{N} 3^{\prime} \ldots \mathrm{S} 1$ & $3.084(7)$ & $\mathrm{N} 1-\mathrm{N} 2-\mathrm{N} 4-\mathrm{N} 5$ & $-7.74(13)$ \\
Fe1-N4 & $1.948(4)$ & $\mathrm{N} 3^{\prime} \ldots \mathrm{S} 2$ & $3.286(14)$ & $\mathrm{C} 1-\mathrm{N} 1-\mathrm{N} 5-\mathrm{C} 20$ & $25.8(3)$ \\
$\mathrm{Fe} 1-\mathrm{N} 5$ & $1.995(3)$ & $\mathrm{N} 1-\mathrm{Fe} 1-\mathrm{N} 2$ & $81.91(17)$ & $\mathrm{N} 2-\mathrm{C} 10-\mathrm{C} 11-\mathrm{N} 4$ & $2.1(3)$ \\
$\mathrm{Fe} 1-\mathrm{N} 6$ & $1.943(4)$ & $\mathrm{N} 2-\mathrm{Fe} 1-\mathrm{N} 4$ & $94.70(16)$ & $\mathrm{C} 20-\mathrm{C} 11-\mathrm{C} 10-\mathrm{C} 1$ & $11.77(7)$ \\
Fe1-N7 & $1.932(4)$ & $\mathrm{N} 4-\mathrm{Fe} 1-\mathrm{N} 5$ & $81.62(17)$ & & \\
$\mathrm{N} 3 \ldots \mathrm{S} 1$ & $3.107(10)$ & $\mathrm{N} 5-\mathrm{Fe} 1-\mathrm{N} 1$ & $102.38(16)$ & & \\
\hline
\end{tabular}




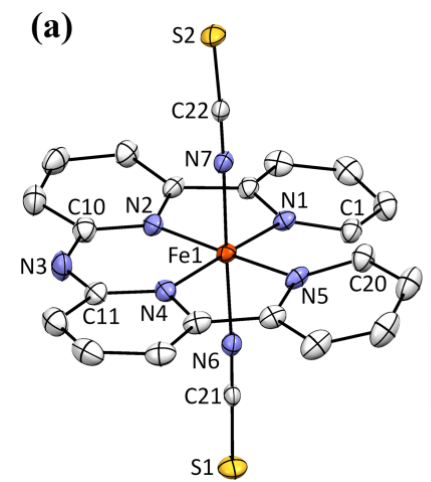

(b)

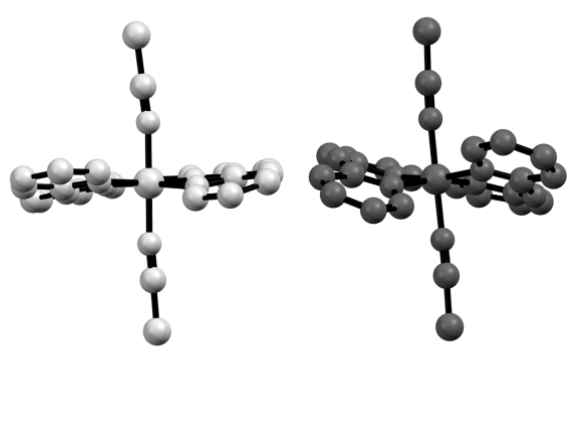

Figure 2. a) Displacement ellipsoid plot (50\% probability level) of compound $\mathbf{1}$ at $110(2) \mathrm{K}$. Hydrogen atoms and disorder are omitted for clarity. b) Side views of $\mathbf{1}$ (light grey) and of $\mathbf{2}^{[17]}$ (dark) in the LS state.

(a)
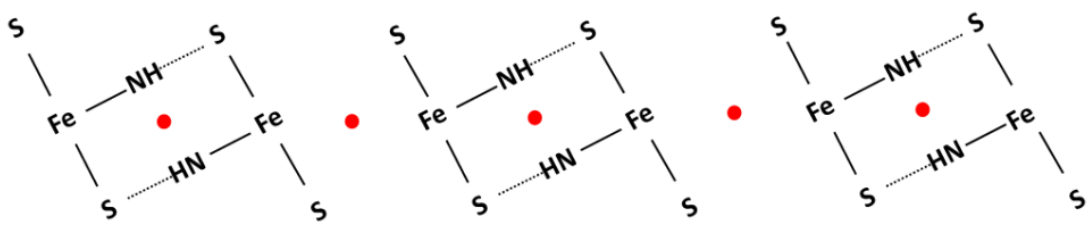

(b)

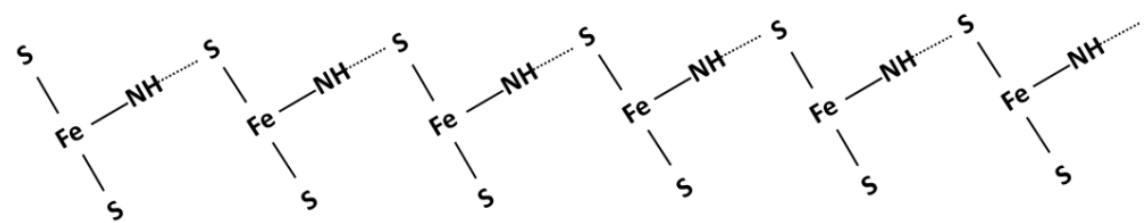

Figure 3. Schematic representations of the two limiting contact modes between adjacent molecules in the crystal packing of $\mathbf{1}$. In mode a, the H-bond network is best described as centrosymmetric $\mathrm{H}$ bond dimers, but there is no propagation of $\mathrm{H}$-bonding interactions in the crystal. In mode $\mathbf{b}$, the propagation of H-bonding interactions is achieved in the crystal. In both modes, only one of the two thiocyanates is $\mathrm{H}$-bond acceptor. The H-bond network in $\mathbf{1}$ is a combination of modes $\mathbf{a}$ and $\mathbf{b}$ (see text). Inversion centers are indicated by dots.

In the structure of $\mathbf{1}$, the Fe complexes are disordered. Disorder may occur during nucleation and crystal growth because of competitive $\mathrm{N}-\mathrm{H} \cdots \mathrm{S}$ hydrogen bond interactions as each molecule has two potential H-bond acceptors (S1 and S2) but only one potential H-bond donor (N3). Optimization of hydrogen bond interactions in the crystal can be achieved via two distinct H-bond networks: (i) centrosymmetric H-bond dimers (mode a) and (ii) one-dimensional (hereafter, 1-D) H-bond chains (mode b) (Figure 3). In mode a, the network is built of hydrogen-bonded dimers with two strong $\mathrm{N}-\mathrm{H} \cdots \mathrm{S}$ hydrogen bonds $\left(\mathrm{N} 3 \cdots \mathrm{S} 2\right.$ and $\left.\mathrm{N} 3^{\prime} \cdots \mathrm{S} 2^{\prime}\right)$. The lack of one extra $\mathrm{N}-\mathrm{H}$ donor in the bbpya ligand prevents the propagation of hydrogen bonds along one direction. In mode $\mathbf{b}$, the network is achieved via 1-D chains featuring two unidirectional hydrogen bonds (N3 '.S 1 ' and $\mathrm{N} 3^{\prime} \cdots \mathrm{S} 1$ ). In both modes, only one thiocyanate is acceptor in one $\mathrm{N}-\mathrm{H} \cdots \mathrm{S}$. The H-bonded network in the structure of $\mathbf{1}$ is a combination of both modes $\mathbf{a}$ and $\mathbf{b}$ so that both thiocyanates can be statistically acceptors in $\mathrm{N}-\mathrm{H} \cdots \mathrm{S}$ hydrogen bonds. Since the Fe complexes are disordered, the propagation of $\mathrm{N}-\mathrm{H} \cdots \mathrm{S}$ hydrogen bonds can be achieved via a set of unidirectional $\mathrm{N}-\mathrm{H} \cdots \mathrm{S}$ hydrogen bonds occurring successively between the major and minor components of the disorder

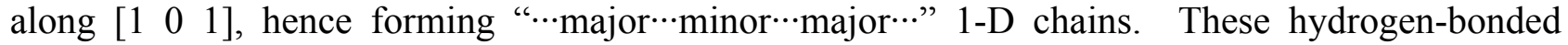
chains have however defects because of the statistical distribution of the molecules over their disordered components. Finally, $\pi-\pi$ stacking interactions also occur in $\mathbf{1}$, as shown by the short distances between the terminal pyridine rings of two neighbouring molecules $\left(\mathrm{Cg} 1{ }^{\cdots}{ }^{\mathrm{Cg}} 2^{\prime}=4.28 \AA\right.$, Figure S3). Overall, in 1 the propagation of strong intermolecular interactions can still be achieved in the long-range order despite the presence of a single $\mathrm{N}-\mathrm{H}$ bridge per molecule. Overall, 
intermolecular interactions observed in the crystal suggest that, if $\mathbf{1}$ has SCO properties, then it may show cooperativity.

To investigate the magnetic properties of 1 the temperature dependence of $\chi_{M} T$ was measured in the range $300-550 \mathrm{~K}$ on a powder sample of $1\left(\chi_{M}\right.$ is the molar magnetic susceptibility and $\mathrm{T}$ the temperature, see Figure $4 \mathrm{a}) \cdot \chi_{M} T$ data show that $\mathbf{1}$ undergoes a complete SCO that, like noncooperative compounds, spans over $150 \mathrm{~K}$, but like strongly cooperative compounds shows a wide $(21 \mathrm{~K})$ hysteretic cycle. At room temperature and up to $c a .330 \mathrm{~K}$ the $\chi_{M} T$ value of $0.01-0.02 \mathrm{~cm}^{3}$ $\mathrm{mol}^{-1} \mathrm{~K}$ is clearly indicative of a LS state, consistent with the dark color of the compound and with the short $\mathrm{Fe}-\mathrm{N}$ distances experimentally determined via X-ray crystallography. Further heating of the sample to $550 \mathrm{~K}$ using an oven was necessary to reach the $\chi_{M} T$ value of $3.02 \mathrm{~cm}^{3} \mathrm{~mol}^{-1} \mathrm{~K}$ expected for an $\mathrm{Fe}(\mathrm{II})$ ion in its $\mathrm{HS}$ state. A strongly dissymmetric hysteresis cycle of $21 \mathrm{~K}$ at its widest point was reproduced over several heating/cooling cycles, showing that it is not the result of irreversible phase transitions or decomposition. The spin crossover occurs at $T_{1 / 2} \uparrow=428(1)$ upon warming and $T_{1 / 2} \downarrow=407(3) \mathrm{K}$ upon cooling, as derived from the maximum of $d\left(\chi_{M} T\right) / d T$ (Figure S4). The average transition temperature $T_{1 / 2}$ is $418 \mathrm{~K}$, and represents one of the highest transition temperatures reported for molecular SCO compounds. ${ }^{[9,10,21-25]}$ Notably it is remarkably higher than that of 2, which, in spite of its similar molecular structure, is HS at room temperature and exhibits a 2 -step transition upon cooling. ${ }^{[17]}$
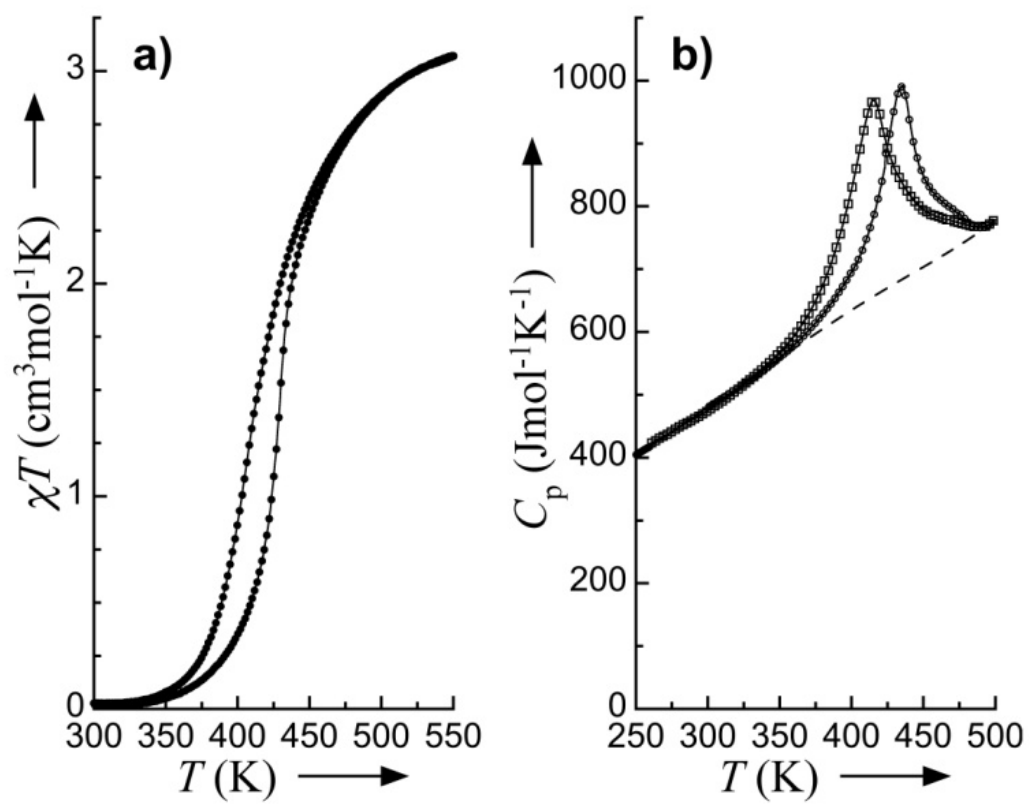

Figure 4. a) Thermal variation of $\chi_{M} T$ for $\mathbf{1}$. b) Molar heat capacities for $\mathbf{1}$ upon warming (empty circles) and cooling (empty squares). The dashed line is the estimated normal heat capacity used for $\Delta C_{p}$ determination. All measurements were performed at $10 \mathrm{~K} \mathrm{~min}^{-1}$.

Attempts of structural characterization of the HS state of 1 were undertaken. Challenges arise as the SCO temperature occurs at a temperature that is not commonly reachable with the temperature controller of most single crystal X-ray diffractometers. However, the HS state could further be characterized by high-temperature powder X-ray diffraction (PXRD) under vacuum (Figure 5). The diffractogram at $553 \mathrm{~K}$ reveals a higher symmetry compared to $297 \mathrm{~K}$, with the peaks at $2 \theta=18.7$, 23.3 and $24.8^{\circ}$ disappearing, and the triplet at $2 \theta=20.3,21.0$, and $21.9^{\circ}$ turning into a more intense doublet at $2 \theta=19.6$ and $20.5^{\circ}$. These changes were reproduced without significant changes over several heating/cooling cycles between $297 \mathrm{~K}$ and $550 \mathrm{~K}$, showing that 1 undergoes a reversible thermal phase transition that must correspond to SCO. 


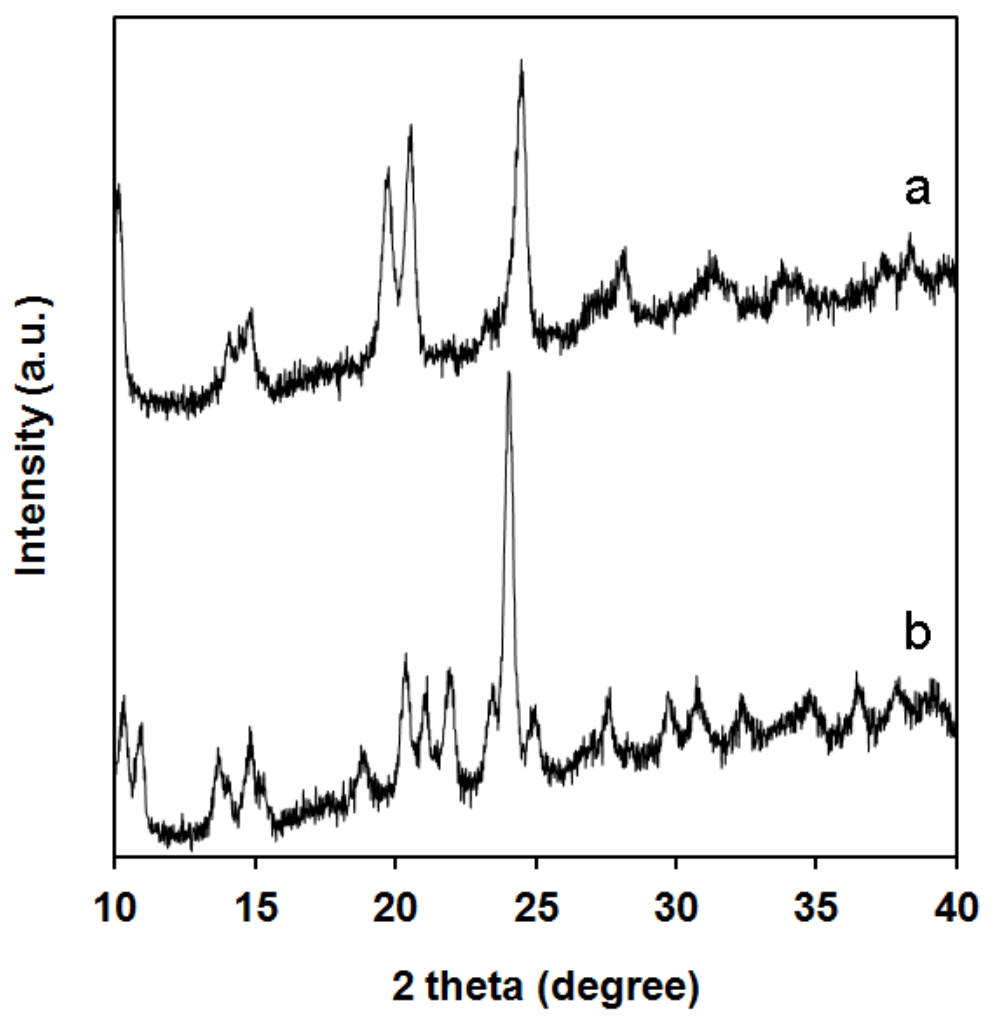

Figure 5. Powder X-ray diffractograms (PXRD) for compound 1 at (a) $553 \mathrm{~K}$, and (b) $297 \mathrm{~K}$. Both PXRD in the heating and cooling modes are identical at $297 \mathrm{~K}$. All PXRD were taken in vacuum $\left(6 \times 10^{-2} \mathrm{mbar}\right)$ to prevent aerial oxidation.

To support these data differential scanning calorimetry (DSC) measurements were undertaken on a powder sample of 1 in the range 150-500 K. The calorimetric data reveal anomalies both in warming and cooling modes at the temperatures $T_{1 / 2}{ }^{\uparrow}=434(1)$ and $T_{1 / 2} \downarrow=415(1) \mathrm{K}$, respectively, as defined by heat capacity maxima (Figure $4 \mathrm{~b}$ ). These temperatures clearly define a hysteresis cycle and match those of the transitions in the $\chi_{M} T$ vs. $T$ plot; they can thus be ascribed to a cooperative SCO phenomenon in 1. The excess enthalpy and entropy associated with the SCO in $\mathbf{1}$ can be derived from integration of $\Delta C_{p}$ (Figure 6a) over $T$ and $\ln T$, respectively, which leads to $\Delta_{\text {sco }} H=$ $12.9 / 15.6 \mathrm{~kJ} \mathrm{~mol}^{-1}$ and $\Delta_{\mathrm{SCO}} S=29.9 / 37.4 \mathrm{~J} \mathrm{~mol}^{-1} \mathrm{~K}^{-1}$ upon warming and cooling, respectively. These relatively large values are in agreement with a cooperative SCO in $\mathbf{1}^{[26,27]}$ To quantify cooperativity the excess heat capacity was fitted to Sorai's domain model (full lines in Figure 6a, see ESI for details). ${ }^{[28]}$ The fit yielded $n=10.2$ and 6.5 upon warming and cooling, respectively, which represents the number of like-spin SCO centers per interacting domain. The former value is consistent with that obtained for the lower temperature transition observed in $\mathbf{2}$, and with the cooperative transition of other reported SCO complexes $\left[\mathrm{Fe}(\mathrm{L})(\mathrm{NCS})_{2}\right]$, where $\mathrm{L}$ is a disubstituted bapbpy derivative. ${ }^{[18]}$ The latter, smaller value is consistent with the more gradual transition / broader excess heat capacity peak observed in other Fe(II) bapbpy-derivative complexes upon cooling. ${ }^{[18]}$ For a direct comparison, HS fractions can be derived from both magnetic and calorimetric data, showing an excellent agreement (Figure 6b). Another quantification of the cooperative character of the SCO in $\mathbf{1}$ can be obtained from these data with the so-called Slichter and Drickamer model. ${ }^{[16]}$ The best agreement is obtained with a mean-field interaction term $\Gamma=$ $8.15 \mathrm{~kJ} \mathrm{~mol}^{-1}$. This result, corresponding to $\Gamma / R T_{1 / 2}=2.34$, is perfectly consistent with the experimental observations, since in this model bistability is predicted for values of $\Gamma / R T_{1 / 2}>2$, and the width of the resulting hysteresis cycle in the SCO curve increases with $\Gamma / R T_{1 / 2}$. Overall, powder $\mathrm{X}$-ray diffraction, magnetic, and calorimetric data provide a consistent view of the spin transition of 1. The observed hysteresis cycle, which is a sign of efficient cooperativity in compound $\mathbf{1}$, is in 
agreement with the intermolecular interactions (H-bonding and $\pi-\pi$ stacking) observed in the crystal packing.
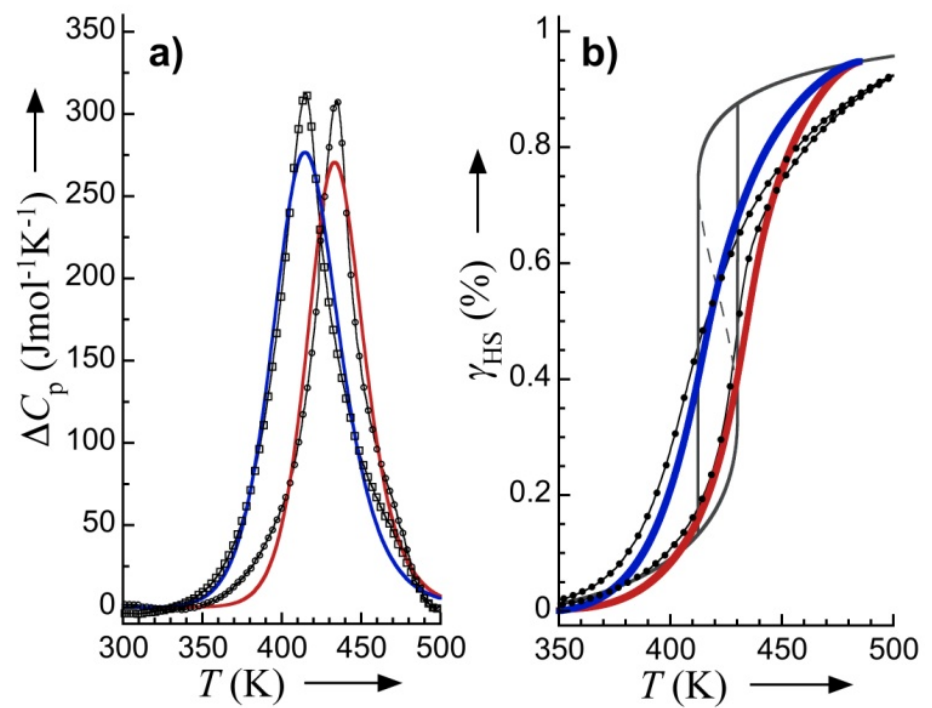

Figure 6. a) Excess molar heat capacities associated with the SCO in 1. Full red and blue lines are fits of the data upon warming (empty circles) and cooling (empty squares) to the domain model of Sorai (see Supporting Information and references 15-16) with $n=10.2$ and 6.5 respectively. b) The spin crossover of 1 shown as the high-spin fraction $\gamma_{\mathrm{HS}}$ vs. $T$ (full red and blue lines are derived from calorimetric data, black dots are from magnetic data), and the simulation with the SlichterDrickamer model (full grey line).

Quantum chemical calculations were realized in order to determine if the difference in transition temperature between $\mathbf{1}$ and $\mathbf{2}$ is due to the different stabilization of the LS state by the bbpya and bapbpy ligand, or due to crystal packing effects that may induce effective pressure shifting $\Delta \mathrm{E}$ and the transition temperature). ${ }^{[29,30]}$ First, DFT geometry optimizations for both complexes were performed in vacuum (see Fe-N distances in Table S1). For the LS state of 2 the Fe-N distances show reasonable agreement with the crystal structure data (LS phase). For 1 a slight deviation was found: the theoretical structure possesses $\mathrm{C}_{2}$ symmetry while the experimental structure slightly deviates from it. For the HS state of $\mathbf{2}$ the geometrical features of the model deviates considerably from the experimental ones, which may be caused by crystal packing effects. For $\mathbf{1}$ the HS crystallographic structure is not available, hence no comparison could be made, but for $\mathbf{2}$ periodic DFT geometry optimizations were performed using the same functional and basis set and keeping the experimental unit cell parameters fixed (see Supplementary Information). The structure optimized in the crystal lattice indeed showed very good agreement with the experimental one (Table S1). Since the geometry optimized in vacuum deviates considerably from the experimental one these results confirm the presence of crystal packing effects in $\mathbf{2}$ that may destabilize the HS state.

Table 2. High spin - low spin energy differences (in $\mathrm{cm}^{-1}$ ) for $\mathbf{1}$ and $\mathbf{2}$ according to DFT and CASPT2 theoretical calculations. ${ }^{\mathrm{a}}$

$\left[\mathrm{Fe}(\right.$ bapbpy $\left.)(\mathrm{NCS})_{2}\right](\mathbf{2})$

$\begin{array}{lccc}\text { Method } & \Delta E_{H L} & \Delta Z P E & \Delta E_{H L}^{0} \\ \text { DFT (PBE) } & 7590 & -768 & 6822 \\ \text { CASPT2 } & -2819 & - & -3587^{\mathrm{b}} \\ \text { Periodic DFT } & 9064 & - & - \\ \text { Single point DFT } & 8792 & - & -\end{array}$


on geometries

optimized in the

crystal

[Fe(bbpya)(NCS) 2$]$ (1)

\begin{tabular}{|c|c|c|c|}
\hline Method & $\Delta E_{H L}$ & $\triangle Z P E$ & $\Delta E_{H L}^{0}$ \\
\hline PBE (PBE) & 9871 & -937 & 8934 \\
\hline CASPT2 & -830 & - & $-1767^{b}$ \\
\hline
\end{tabular}

The HS - LS energy differences $\Delta \mathrm{E}_{\mathrm{HL}}^{0}$ were also calculated using the DFT-minimized structures (Table 2). The zero-point vibrational corrections $\triangle \mathrm{ZPE}$ to the total energy were found in the range of -700 to $-1000 \mathrm{~cm}-1$ (Table 2), which is typical for spin-crossover materials. ${ }^{[31,32]}$ However, the absolute values of the DFT (PBE) energy differences $\Delta \mathrm{E}_{\mathrm{HL}}$ were found in the range of $7000-$ $10000 \mathrm{~cm}^{-1}$ which is way too high for SCO compounds with thermal spin crossover and would result in transition temperatures around $1000 \mathrm{~K}$ and higher (see for example $\left[\mathrm{Fe}(\text { terpy })_{2}\right]^{2+}$ in reference ${ }^{[31]}$ ). Periodically optimized structures (for 2) show that the HS - LS energy difference is about $1500 \mathrm{~cm}^{-1}$ larger compared to that in vacuum. This result again confirmed the presence of crystal packing effects for complex 2 , but did not really fit to the experimental values of the transition temperatures found for $\mathbf{1}$ and $\mathbf{2}$. Single point DFT calculations performed on geometries of the complexes optimized in the crystal showed a similar HS - LS energy difference which indicates that the crystal packing influences the HS - LS energy difference mainly through modification of the geometry. Upon going from the vacuum optimized geometries to the geometries optimized in the crystal structure, the total energy of the LS state complex is destabilized less than that of the HS state geometry, resulting in the larger $\Delta E_{H L}$ for the crystal structures. The most pronounced changes in the HS state geometry by crystal packing are elongations of the Fe-NCS bonds and shortenings of the Fe-N1 bonds (See table S1 and the geometries of the corresponding structures in the supplementary material). This modification of the geometry also results in moderate changes of the orbital energies, although, due to severe mixing of the iron d-orbitals with the ligand orbitals, it is difficult to conclusively say how these changes in orbital energies of the $t_{2 g}$ and $\mathrm{e}_{\mathrm{g}}$-like iron d-orbitals affect the total energy difference.

Whereas the geometry and vibrational frequencies of SCO complexes are usually well reproduced by DFT, the accurate determination of the HS - LS energy difference is indeed a well-known problem of DFT, as this method is a single reference method incapable of a proper description of the multi-configurational nature of the spin states unless the functional is empirically reparametrized. ${ }^{[33,34]}$ In order to get accurate HS-LS energy differences $\Delta E_{H L}^{0}$ one has to use ab initio methods such as CASSCF/CASPT2, which is much more computationally demanding and typically does not allow for the full geometry optimization for SCO materials, all the more in a crystal lattice. For this reason CASPT2 energy calculations were performed on the DFT geometries optimized in vacuum; the energies are presented in Table 2 as well. The $\Delta E_{H L}$ and $\Delta E_{H L}^{0}$ values found for $\mathbf{1}$ and $\mathbf{2}$ were negative, which is also in disagreement with the experiments, as it would indicate that both complexes should remain in the HS state. However, these values confirmed the DFT results, as for complex 1 the HS - LS energy difference was about $2000 \mathrm{~cm}^{-1}$ larger than for $\mathbf{2}$. Overall, the fact that the energy difference $\Delta E_{H L}^{0}$ between the LS and HS state in vacuum for compound $\mathbf{1}$ was found about $2000 \mathrm{~cm}^{-1}$ larger than for compound $\mathbf{2}$ with both methods confirms that the stronger ligand field generated by bbpya around iron(II) stabilizes the LS state and destabilizes the HS state, compared to bapbpy. Thus, the bbpya ligand must play a significant contribution in the increase of the transition temperature for $\mathbf{1}$, compared to $\mathbf{2}$. Secondly, periodic DFT calculations show that 
crystal-packing effects are significant for compound $\mathbf{2}$ where they result in the destabilization of the HS state by about $1500 \mathrm{~cm}^{-1}$. We may speculate that similar effects are present for 1 , but the absence of the crystal structure of the HS phase has prevented us, up to now, from performing similar calculations.

\section{Conclusion}

Ligand design aiming at replacing one 6-membered ring in $\mathbf{2}$ by a 5 -membered ring to increase the transition temperature of the SCO, led to the new bis-bipyridine ligand bbpya and its iron complex 1. Compound 1 not only shows SCO properties, but its transition temperature is remarkably enhanced (by ca. $200 \mathrm{~K}$ ) compared to 2 . Hence, the LS state remains the most stable state of $\mathbf{1}$ at room temperature, whereas $\mathbf{2}$ is HS at room temperature. Thus, the bbpya ligand offers a unique opportunity to stabilize the LS state of iron(II) complexes, as confirmed by DFT and CASPT2 calculations. In addition, although the number of N-H bridges per molecule of bbpya is reduced by a factor of 2 compared to bapbpy, disorder in $\mathbf{1}$ facilitates the propagation of $\mathrm{H}$-bonding intermolecular interactions in the crystal lattice, thus forming disordered infinite supramolecular chains in the solid. Overall, 1 not only shows one of the highest transition temperatures recorded among mononuclear SCO complexes, but it keeps a large (dissymmetric) hysteresis cycle of $21 \mathrm{~K}$. On the one hand, these results suggest that hydrogen bonds can contribute propagating intermolecular interactions leading to hysteresis in high-temperature molecular SCO materials. On the other hand, they open a unique opportunity to study the mechanism of cooperative SCO in molecular compounds, as many experimental techniques (e.g., near-field or optical microscopy) are easier to implement upon heating the sample above room temperature, than upon cooling at cryogenic temperatures.

\section{Experimental part}

All chemicals were obtained from commercial sources and used without further purification, unless otherwise mentioned. 6-Bromo-2,2'-bipyridine was purchased from Sigma-Aldrich (CAS 1049573-5), and 6-amino-2,2'-bipyridine was synthesized according to previously reported procedures. ${ }^{[19 \text {, }}$ ${ }^{20]}$ Experiments that needed an inert environment were performed using standard Schlenk line techniques. The applied vacuum was about $10^{-3}$ mbar. Degassed solvents were obtained by bubbling argon through the solvent in a Schlenk flask for at least one hour. For all ligand and complex syntheses, degassed solvents were used; for ligand purifications solvents were used without further purification. Filtration of complexes was carried out using Whatman RC 60 membrane filters. For other filtrations Whatman 597 filters were used. NMR Spectra were measured on a Bruker DPX-300 Spectrometer at room temperature. Mass spectra were obtained using soft electron spray from a Thermoquest Finnagen AQA. High-resolution mass spectra were measured using direct injection $(2 \mu \mathrm{L}$ of a $2 \mu \mathrm{M}$ solution in DMSO on a (Thermo Finnigan LTQ Orbitrap) mass spectrometer equipped with an electron spray ion source in positive mode (source voltage $3.5 \mathrm{kB}$, sheath gas flow 10 , capillary temperature $275^{\circ} \mathrm{C}$ ) with resolution $\mathrm{R}=60.000$ at $\mathrm{m} / z$ $=400$ (mass range $=150-2000)$ and dioctylphtalate $(\mathrm{m} / \mathrm{z}=391.28428)$ as "lock mass". IR spectra were acquired on a PerkinElmer FT-IR Spectrometer Spectrum Two at room temperature. Elemental analysis $(\mathrm{C}, \mathrm{H}, \mathrm{N}, \mathrm{S})$ were obtained from a Perkin-Elmer 2400 Series II analyzer.

Synthesis of bbpya. A mixture of 6-bromo-2,2'-bipyridine $(250 \mathrm{mg}, 1.07 \mathrm{mmol}),\left[\mathrm{Pd}(\mathrm{dba})_{2}\right](13$ $\mathrm{mg}, 0.022 \mathrm{mmol}$ ), (S)-BINAP (27 mg, $0.043 \mathrm{mmol})$ and $\mathrm{KO} t \mathrm{Bu}(483 \mathrm{mg}, 4.30 \mathrm{mmol})$ was put under argon and partially dissolved in degassed toluene $(20 \mathrm{~mL})$ in a dry round-bottom flask. The mixture was stirred for $10 \mathrm{~min}$ and 6-amino-2,2'-bipyridine $(220 \mathrm{mg}, 1.28 \mathrm{mmol})$ was added, followed by heating the reaction mixture to $80{ }^{\circ} \mathrm{C}$. After 3 days, the brown mixture was cooled down with an ice bath. Deionized water $(25 \mathrm{~mL})$ was added and the mixture was stirred for 1 hour. No solid appeared and the resultant mixture was then extracted with DCM $(3 \times 40 \mathrm{~mL})$. The 
organic layers were combined and dried over $\mathrm{MgSO}_{4}$, followed by filtration to remove $\mathrm{MgSO}_{4}$ and evaporation of solvent DCM under reduced pressure, giving a brown oil. Adding cold $\mathrm{MeOH}$ to the oil resulted in the formation of solids which were filtered, washed with cold $\mathrm{MeOH}$ and dried under high vacuum, to afford bbpya as a white solid (241 mg, 69\%). $\mathrm{R}_{\mathrm{f}}=0.09\left(\mathrm{SiO}_{2}: \mathrm{MeOH} / \mathrm{DCM}: 1 / 9\right)$. ${ }^{1} \mathrm{H}$ NMR $\left(\mathrm{DMSO}-d^{6}\right): \delta=9.90(\mathrm{~s}, 1 \mathrm{H}, \mathrm{NH}), 8.69(\mathrm{ddd}, J=4.8,1.9,0.9 \mathrm{~Hz}, 2 \mathrm{H}, \mathrm{H}-7), 8.38(\mathrm{dt}, J=$ 8.0, 1.1 Hz, 2H, H-4), 7.99 (td, $J=7.7,1.8 \mathrm{~Hz}, 2 \mathrm{H}, \mathrm{H}-6), 7.93$ (dt, $J=5.4,2.7 \mathrm{~Hz}, 2 \mathrm{H}, \mathrm{H}-3), 7.89$ $(\mathrm{d}, J=2.9 \mathrm{~Hz}, 4 \mathrm{H}, \mathrm{H}-1, \mathrm{H}-2), 7.45$ (ddd, $J=7.5,4.7,1.2 \mathrm{~Hz}, 2 \mathrm{H}, \mathrm{H}-5) .{ }^{13} \mathrm{C}$ NMR (DMSO- $\left.d^{6}\right): \delta=$ $155.5(\mathrm{Cq}), 153.9(\mathrm{Cq}), 153.4(\mathrm{Cq}), 149.3$ (C-7), 138.9 (C-1), 137.3 (C-6), 124.0 (C-5), 120.4 (C-4), $112.8(\mathrm{C}-3), 112.3(\mathrm{C}-2) \mathrm{ppm} .{ }^{1} \mathrm{H}$ NMR $\left(\mathrm{CD}_{2} \mathrm{Cl}_{2}\right): \delta=8.72-8.61(\mathrm{~m}, 2 \mathrm{H}), 8.40(\mathrm{~d}, J=8.0 \mathrm{~Hz}$, $2 \mathrm{H}), 8.07-7.97(\mathrm{~m}, 2 \mathrm{H}), 7.90-7.77(\mathrm{~m}, 4 \mathrm{H}), 7.71(\mathrm{~d}, J=8.2 \mathrm{~Hz}, 2 \mathrm{H}), 7.54(\mathrm{~s}, 1 \mathrm{H}), 7.32(\mathrm{ddd}, J=$ 7.4, 4.7, $1.1 \mathrm{~Hz}, 2 \mathrm{H})$. IR spectroscopy $\left(\mathrm{cm}^{-1}\right): 3390,2922,2852,1582,1558,1520,1472,1447$, 1418, 1340, 1296, 1271, 1230, 1152, 1091, 1073, 1050, 990, 963, 902, 818, 774, 738, 679, 668, 644, 620, 572, 402, 341, 317. ES-MS (MeOH) $\mathrm{m} / z$ (calc): $326.1\left(326.4,[M+\mathrm{H}]^{+}\right), 348.0(348.4$, $\left.[M+\mathrm{Na}]^{+}\right), 673.2\left(673.7,[2 M+\mathrm{Na}]^{+}\right)$. Elemental analysis calcd $(\%)$ for $\mathrm{C}_{20} \mathrm{H}_{15} \mathrm{~N}_{5}: \mathrm{C} 73.82, \mathrm{H} 4.65$, N 21.53; found: C 73.21, H 5.36, N 20.39.

Preparation of [Fe(bbpya)(NCS $)_{2}$ ] (1). First, an $\mathrm{Fe}(\mathrm{NCS})_{2}$ solution was prepared by weighing KSCN (195 mg, $2.00 \mathrm{mmol})$ and ascorbic acid $(6.1 \mathrm{mg}, 0.035 \mathrm{mmol})$ under argon into a roundbottom flask. $\mathrm{FeSO}_{4}(152 \mathrm{mg}, 1.00 \mathrm{mmol})$ was added and the mixture was suspended in degassed methanol $(6.0 \mathrm{~mL})$. The suspension was stirred for $40 \mathrm{~min}$, followed by filtration and the filtrate was transferred into a volumetric flask. The volume was adjusted to $10.0 \mathrm{~mL}$ with degassed methanol and the volumetric flask was well shaken, yielding a $0.1 \mathrm{M}\left[\mathrm{Fe}(\mathrm{NCS})_{2}\right]$ solution in methanol. Since the filtration and the iron solution were not kept under argon, the iron solution had to be prepared fresh for every synthesis. The oxidation of the iron solution was visible by a change of colour (from colourless to dark violet).

Then, the ligand bbpya (30 mg, $0.093 \mathrm{mmol})$ was dissolved in degassed methanol (3 $\mathrm{mL})$ in a round-bottom flask and stirred under argon, to give a yellow solution. $1.1 \mathrm{Eq}$. of the $0.1 \mathrm{M}$ iron solution $(0.1 \mathrm{mmol})$ was added to the dissolved ligand, resulting in a purple suspension. The mixture was stirred 16 hours under argon. The purple solid was collected by filtration and washed with degassed methanol $(3 \times 5 \mathrm{~mL})$. The purple solid was dried under high vacuum for 3 hours $(31$ $\mathrm{mg}, 67 \%)$. IR spectroscopy $\left(\mathrm{cm}^{-1}\right): 3269,3184,3130,3099,3047,2125\left(\mathrm{NCS}^{-}\right), 2109\left(\mathrm{NCS}^{-}\right)$, 1625, 1601, 1582, 1529, 1478, 1464, 1451, 1416, 1406, 1302, 1250, 1172, 1164, 1139, 1090, 1022, $949,870,800,759,725,685,661,643,629,495,478$. HR-MS (DMSO): $\mathrm{m} / z$ (calc): 497.0170 (497.0174, $\left.[M]^{+}\right), 439.0420\left(439.0423,[M-\mathrm{NCS}]^{+}\right) .{ }^{1} \mathrm{H}$ NMR (DMSO- $\left.d^{6}\right): \delta=9.92(\mathrm{~s}, 1 \mathrm{H}), 8.68(\mathrm{~d}$, $J=3.9,2 \mathrm{H}), 8.38(\mathrm{~d}, J=7.7,2 \mathrm{H}), 8.06-7.80(\mathrm{~m}, 6 \mathrm{H}), 7.46(\mathrm{dd}, J=6.8,4.7,2 \mathrm{H}), 6.34(\mathrm{~s}, 1 \mathrm{H})$. Integration of the peaks was difficult due to the low solubility of the complex. Elemental analysis calcd (\%) for $\mathrm{C}_{22} \mathrm{H}_{15} \mathrm{FeN}_{7} \mathrm{~S}_{2}$ : C 53.13, H 3.04, N 19.73, S 12.87; found: C 52.56, H 2.93, N 19.73, S 12.77 .

Single crystals growing for 1 . The ligand bbpya $(15 \mathrm{mg})$ was dissolved in degassed DMF (3 $\mathrm{mL})$ to afford a clear yellow solution. A small amount of ascorbic acid (5 mg) was added to prevent aerial oxidation. $1 \mathrm{~mL}$ aliquots of this solution were pipette-filtered over $1 \mathrm{~cm}$ Celite into the vertical compartment of a Y-shape glass tube, $\mathrm{Fe}(\mathrm{NCS})_{2}(0.5 \mathrm{~mL}$ of a $0.1 \mathrm{M}$ solution) was carefully added into the other compartment of the glass tube. Liquid nitrogen was used to freeze both compartments before degassed $\mathrm{MeOH}$ was added to fill the Y-shaped tubes. Each tube was then stoppered and kept at room temperature under argon using balloons, if possible in a sunny place as light seems to play a role in the crystallization. Single crystals were obtained within a week. Yield: $90 \%$. The crystals were not air-sensitive and did not indicate loss of solvent therefore could be handled out of the mother liquor and at ambient conditions for several weeks.

Crystal data. $\mathrm{FW}=497.38$, black irregular lath, $0.43 \times 0.12 \times 0.08 \mathrm{~mm}^{3}$, triclinic, $P-1$ (no. 2 ), $a=$ 8.8024(4), $b=8.8862(4), c=13.7229(5) \AA, \alpha=100.637(3), \beta=103.916(3), \gamma=95.972(3)^{\circ}, V=$ 1011.52(8) $\AA^{3}, Z=2, D_{\mathrm{x}}=1.633 \mathrm{~g} \mathrm{~cm}^{-3}, \mu=8.134 \mathrm{~mm}^{-1}$, abs. corr. range: $0.195-0.596 .12241$ Reflections were measured up to a resolution of $(\sin \theta / \lambda)_{\max }=0.62 \AA^{-1} .3945$ Reflections were unique $\left(R_{\text {int }}=0.0215\right)$, of which 3502 were observed $[\mathrm{I}>2 \sigma(\mathrm{I})] .578$ Parameters were refined using 
1152 restraints. $R 1 / w R 2$ [I $>2 \sigma(\mathrm{I})$ ]: 0.0294/0.0796. $R 1 / w R 2$ [all refl.]: $0.0335 / 0.0830 . \mathrm{S}=1.074$. Residual electron density found between -0.29 and 0.24 e $\AA^{-3}$.

Details of single crystal X-ray structure determination. All reflection intensities were measured at $110(2) \mathrm{K}$ using a SuperNova diffractometer (equipped with Atlas detector) with $\mathrm{Cu} K \alpha$ radiation $(\lambda=1.54178 \AA$ ) under the program CrysAlisPro (Version 1.171.36.28 Agilent Technologies, 2013). The program CrysAlisPro was used to refine the cell dimensions and for data reduction.. The structure was solved with the program SHELXS-2013 ${ }^{[35]}$ and was refined on $F^{2}$ with SHELXL2013. ${ }^{[35]}$ Analytical numeric absorption corrections based on a multifaceted crystal model were applied using CrysAlisPro. The temperature of the data collection was controlled using the system Cryojet (manufactured by Oxford Instruments). The $\mathrm{H}$ atoms were placed at calculated positions using the instructions AFIX 43 with isotropic displacement parameters having values 1.2 times Ueq of the attached $\mathrm{C}$ or $\mathrm{N}$ atoms. The Fe complex is disordered over two orientations, and both components of the disorder are related by a pseudo inversion center. The occupancy factor of the major component of the disorder refines to $0.7038(15)$. In order to keep the data-to-parameter ratio to an acceptable level, the EADP constraints were used for all related disordered atoms related via inversion symmetry (no EADP constraints were applied to the heaviest atoms Fe and S).

Additional note: The structure was initially solved in the space group $P 1$ (no. 1) with $Z^{\prime}=2$. However, the ADDSYM procedure in PLATON (A.L.Spek, Acta Cryst. 2009, D65, 148-155) suggested a missed inversion center and the space group $P-1$ (no. 2). Another warning sign for choosing the wrong space group $P 1$ was the value of the Flack parameter (H. D. Flack, Acta Cryst, 1983, $A 39,876-881$ ) that refined exactly to 0.5 . A refinement using a racemic twin model with the transformation matric (-1 $00 / 0-10 / 00-1)$ did not improve the model much, and the structure remained significantly disordered in $P 1$. Additional check for twinning was done using the TwinRotMat procedure in PLATON, but no twin law could be detected. Eventually, the structure was solved and refined in the space group $P-1$ (no. 2).

Magnetic susceptibility measurements. Magnetic measurements were performed on a powder sample 1 using the VSM-oven option of a Quantum Design PPMS set-up of the Physical Measurments unit of the Servicio General de Apoyo a la Invesitigación-SAI, Universidad de Zaragoza. The powder was pressed into $3 \mathrm{~mm}$ diameter pellets of 3.1 and $2.8 \mathrm{mg}$ for the two sets of measurements, performed to verify the reproducibility. The DC magnetization was determined in an applied field of $5 \mathrm{~T}$, and the scan rate was $10 \mathrm{~K} / \mathrm{min}$, the smallest allowed by the set-up. Several warming-cooling scans were performed, showing only little variation between the first and second scan. The data reported here correspond to the third stable and reproducible cycle of measurement. Corrections for the diamagnetism of the sample were calculated using Pascal's constant. ${ }^{[36]}$

Differential Scanning Calorimetry. DSC measurements were performed with a Q1000 calorimeter from TA Instruments equipped with the LNCS accessory. The temperature and enthalpy scales were calibrated with a standard sample of indium, using its melting transition $\left(156.6{ }^{\circ} \mathrm{C}\right.$, $3296 \mathrm{~J} \mathrm{~mol}^{-1}$ ). The measurements were carried out using aluminium pans with a mechanical crimp, with an empty pan as reference. The zero-heat flow procedure described by TA Instruments was followed to derive heat capacities, using a synthetic sapphire as reference compound. An overall accuracy of ca. $0.2 \mathrm{~K}$ for the temperature and up to 5 to $10 \%$ for the heat capacity was estimated over the whole temperature range, by comparison with the synthetic sapphire. A lattice heat capacity was estimated from data below and above the anomaly associated with the SCO process (dashed line in Figure 4b). Excess enthalpy and entropy were derived by integration of the excess heat capacity with respect to $T$ and $\ln T$, respectively.

Modelling magnetic and calorimetric data with the domain model and the Slichter-Drickamer model. The phenomenological domain model developed by Sorai ${ }^{[26,27]}$ was applied, as it is widely used to analyse the SCO behaviour in cases where calorimetric data are available. It is based on heterophase fluctuations and gives a measure of cooperativity through the number of like-spin molecules (or here the SCO centres) $n$ per interacting domain, the larger the domain the more cooperative the transition. According to this model, the heat capacity variation can be written as in Equation 1: 


$$
\Delta C_{p}=\frac{n\left(\Delta_{S C O} H\right)^{2}}{R T^{2}} \frac{\exp \left[\frac{n \Delta_{S C O} H}{R}\left(\frac{1}{T}-\frac{1}{T_{1 / 2}}\right)\right]}{\left\{1+\exp \left[\frac{n \Delta_{S C O} H}{R}\left(\frac{1}{T}-\frac{1}{T_{1 / 2}}\right)\right]\right\}^{2}}
$$

(Equation 1)

The experimental heat capacity data were thus fitted to Equation 1 using $\Delta_{S C O} H$ as derived from integration of $\Delta C_{p}$ vs. $T$, giving $n=10.2 / 6.5$ and $T_{1 / 2}=434 / 415 \mathrm{~K}$ upon warming and cooling, respectively. For $n=1$ the model is equivalent to a pure solution behaviour (van't Hoff equation) with no cooperative effects.

A simple phenomenological expression (see Equation 2) derived from the free energy of a regular solid solution of HS and LS molecules with an interaction term according to the mean-field theory, first used by Slichter and Drickamer, ${ }^{[37]}$ reproduces well the different forms of SCO curves $\left(\gamma_{\mathrm{HS}} v s\right.$. $T$, where $\gamma_{\mathrm{HS}}$ is the fraction of HS species) and also the hysteresis effect for sufficiently large values of the interaction parameter $\Gamma$. With the goal of attaining a mean-field estimation of cooperativity in the material under study, the experimental HS fraction calculated from magnetic measurements were fitted to Equation 2, fixing the thermodynamic figures to the ones determined by DSC calorimetry.

$$
\ln \left(\frac{1-\gamma_{H S}}{\gamma_{H S}}\right)=\frac{\Delta_{S C O} H+\Gamma\left(1-2 \gamma_{H S}\right)}{R T}-\frac{\Delta_{S C O} S}{R}
$$

(Equation 2)

Because Equation 2 can only account for the amplitude of a hysteresis loop and not for its shape, it was considered that the vertical tangents of the calculated S-curve must correspond to $T_{1 / 2} \uparrow$ and $T_{1 / 2 \downarrow} \downarrow$.

The HS fraction $\gamma_{\mathrm{HS}}$ was deduced from the magnetic data using the relation $\gamma_{\mathrm{HS}}(T)=\left(\chi_{M} T-\chi_{M} T_{\mathrm{LS}}\right) /$ $\left(\chi_{M} T_{\mathrm{HS}}-\chi_{M} T_{\mathrm{LS}}\right)$, where $\chi_{M} T_{\mathrm{LS}}$ and $\chi_{M} T_{\mathrm{HS}}$ stand respectively for the values of $\chi_{M} T$ in the LS and HS states. Values of 0.01 and $3.25 \mathrm{~cm}^{3} \mathrm{~mol}^{-1} \mathrm{~K}$ were considered respectively.

The HS fraction $\gamma_{\mathrm{HS}}$ was deduced from the calorimetric data using the relation $\gamma_{\mathrm{HS}}(T)=\Delta H / \Delta_{S C O} H$ where $\Delta_{S C O} H$ is the value derived by integration of the excess heat capacity vs. $T$, multiplied by 1.05 to take into account the likely underestimation associated with few data above the heat capacity anomaly.

Computational details. Vacuum and periodic density functional theory geometry optimizations and frequency calculations for both complexes have been performed using the PBE functional ${ }^{[38]}$ and POB-DZP basis set. ${ }^{[38]}$ Vacuum calculations have been performed with the Turbomole 6.4 package $^{[39,40]}$ while periodic calculations have been done using Crystall4 (a shrinking factor of 4 was used for the k-point mesh; the LS state spacegroup was P-1 and the HS state spacegroup C2/c). ${ }^{[41]}$ Single point CASPT2 calculations were performed for the vacuum DFT optimised structures of the complexes. For the CASPT2 calculations, the active space consisted of 10 electrons distributed over 12 orbitals which included the five metal-centered $3 \mathrm{~d}$ and $3 \mathrm{~d}^{\prime}$ orbitals and two ligand $\sigma$-orbitals. ${ }^{[42,43]}$ The CASPT2 calculations were performed using MOLCAS $7.4 .^{[44,45]}$ Scalar relativistic effects were included using a Douglas-Kroll-Hess Hamiltonian and the basis set used in the calculations was the ANO-RCC basis set that is designed to include relativistic effects. $^{[46,47]}$ The contracted Gaussian basis functions are $(7 \mathrm{~s}, 6 \mathrm{p}, 5 \mathrm{~d}, 4 \mathrm{f}, 3 \mathrm{~g}, 2 \mathrm{~h})$ for Fe, (4s, 3p, 1d) for $\mathrm{N}$ atoms bonded to $\mathrm{Fe},(3 \mathrm{~s}, 2 \mathrm{p})$ for the remaining $\mathrm{N}$ atoms and $\mathrm{C}$ atoms, $(4 \mathrm{~s}, 3 \mathrm{p})$ for $\mathrm{S}$ and $(2 \mathrm{~s})$ for $\mathrm{H}$.

\section{Ackowledgements}

The COST action CM1305 ECOSTBio is acknowledged for stimulating scientific discussion.

\section{Supplementary Information available}


Powder X-ray diffractograms and infrared spectra at room temperature for $\mathbf{1}$, intermolecular distances in the crystal structure of $\mathbf{1}$ (LS phase), derivative of $\chi_{M} T$ versus $T$ plot for complex 1, and bond distances and angles of the minimized structures, are provided in the Supplementary Information. 


\section{References}

[1] P. Gütlich and H. A. Goodwin, Top. Curr. Chem., 2004, 233, 1-47.

[2] M. A. Halcrow, Chem. Soc. Rev., 2011, 40, 4119-4142.

[3] H. Spiering, T. Kohlhaas, N. Romstedt, A. Hauser, C. Bruns-Yilmaz, J. Kusz and P. Gütlich, Coord. Chem. Rev., 1999, 192, 629-647.

[4] O. Kahn and C. J. Martinez, Science, 1998, 279, 44-48.

[5] B. Schafer, C. Rajnak, I. Salitros, O. Fuhr, D. Klar, C. Schmitz-Antoniak, E. Weschke, H. Wende and M. Ruben, Chem. Commun., 2013, 49, 10986-10988.

[6] O. Roubeau, Chem. Eur. J., 2012, 18, 15230-15244.

[7] M. C. Muñoz and J. A. Real, Coord. Chem. Rev., 2011, 255, 2068-2093.

[8] Y. Raza, F. Volatron, S. Moldovan, O. Ersen, V. Huc, C. Martini, F. Brisset, A. Gloter, O. Stephan, A. Bousseksou, L. Catala and T. Mallah, Chem. Commun., 2011, 47, 11501-11503.

[9] R. Boca, M. Boca, L. Dlhan, K. Falk, H. Fuess, W. Haase, R. Jarošciak, B. Papankova, F. Renz and M. Vrbova, Inorg. Chem., 2001, 40, 3025-3033.

[10] I. Šalitroš, N. T. Madhu, R. Boča, J. Pavlik and M. Ruben, Monatsh. Chem., 2009, 140, 695733.

[11] L. Zhang, G.-C. Xu, H.-B. Xu, T. Zhang, Z.-M. Wang, M. Yuan and S. Gao, Chem. Commun., 2010, 46, 2554-2556.

[12] B. Weber, W. Bauer and J. Obel, Angew. Chem. Int. Ed., 2008, 47, 10098-10101.

[13] P. Guionneau, M. Marchivie, G. Bravic, J.-F. Létard and D. Chasseau, Top. Curr. Chem., 2004, 234, 97-128.

[14] B. Weber, W. Bauer, T. Pfaffeneder, M. M. Dîrtu, A. D. Naik, A. Rotaru and Y. Garcia, Eur. J. Inorg. Chem., 2011, 21, 3193-3206.

[15] M. A. Halcrow, Chem. Lett., 2014, 43, 1178-1188.

[16] C. P. Slichter and H. G. Drickamer, J. Chem. Phys., 1972, 56, 2142-2160.

[17] S. Bonnet, M. A. Siegler, J. S. Costa, G. Molnar, A. Bousseksou, A. L. Spek, P. Gamez and J. Reedijk, Chem. Commun., 2008, 5619-5621.

[18] Z. Arcis-Castíllo, S. Zheng, M. A. Siegler, O. Roubeau, S. Bedoui and S. Bonnet, Chem. Eur. J., 2011, 17, 14826-14836.

[19] T. Norrby, A. Börje, L. Zhang and B. Åkermark, Acta Chem. Scand., 1998, 52, 77-85.

[20] J. Yin, B. Xiang, M. A. Huffman, C. E. Raab and I. W. Davies, J. Org. Chem., 2007, 72, 45544557.

[21] X. Bao, P.-H. Guo, W. Liu, J. Tucek, W.-X. Zhang, J.-D. Leng, X.-M. Chen, I. y. Gural'skiy, L. Salmon, A. Bousseksou and M.-L. Tong, Chem. Sci., 2012, 3, 1629-1633.

[22] M. B. Bushuev, V. A. Daletsky, D. P. Pishchur, Y. V. Gatilov, I. V. Korolkov, E. B. Nikolaenkova and V. P. Krivopalov, Dalton Trans., 2014, 43, 3906-3910.

[23] G. Schwarz, Y. Bodenthin, Z. Tomkowicz, W. Haase, T. Geue, J. Kohlbrecher, U. Pietsch and D. G. Kurth, J. Am. Chem. Soc., 2010, 133, 547-558.

[24] G. S. Matouzenko, S. A. Borshch, E. Jeanneau and M. B. Bushuev, Chem. Eur. J., 2008, 15, 1252-1260.

[25] R. Boca, F. Renz, M. Boča, H. Fuess, W. Haase, G. Kickelbick, W. Linert and M. VrbováSchikora, Inorg. Chem. Commun., 2005, 8, 227-230.

[26] M. Sorai and S. Seki, J. Phys. Chem. Solids, 1974, 35, 555-570.

[27] M. Sorai, Top. Curr. Chem., 2004, 235, 153-170.

[28] M. Sorai, Y. Nakazawa, M. Nakano and Y. Miyazaki, Chem. Rev., 2012, 113, PR41-PR122.

[29] A. Hauser, C. Enachescu, M. L. Daku, A. Vargas and N. Amstutz, Coord. Chem. Rev., 2006, $250,1642-1652$.

[30] A. Vef, U. Manthe, P. Gutlich and A. Hauser, J. Chem. Phys., 1994, 101, 9326-9332.

[31] A. Rudavskyi, C. Sousa, C. de Graaf, R. W. A. Havenith and R. Broer, J. Chem. Phys., 2014, 140 , 
[32] M. Gruden-Pavlović, S. Stepanović, M. Perić, M. Güell and M. Swart, Phys. Chem. Chem. Phys., 2014, 16, 14514-14522.

[33] O. Salomon, M. Reiher and B. A. Hess, J. Chem. Phys., 2002, 117, 4729-4737.

[34] M. Reiher, O. Salomon and B. A. Hess, Theor. Chem. Acc., 2001, 107, 48-55.

[35] G. M. Sheldrick and T. R. Schneider in [16] SHELXL: High-resolution refinement, Vol. 277 Academic Press, 1997, pp. 319-343.

[36] G. A. Bain and J. F. Berry, J. Chem. Educ., 2008, 85, 532-536.

[37] C. P. Slichter and H. Drickame, J. Chem. Phys., 1972, 56, 2142-2161.

[38] J. P. Perdew, K. Burke and M. Ernzerhof, Phys. Rev. Lett., 1996, 77, 3865-3868.

[39] O. Treutler and R. Ahlrichs, J. Chem. Phys., 1995, 102, 346-354.

[40] M. von Arnim and R. Ahlrichs, J. Comput. Chem., 1998, 19, 1746-1757.

[41] R. Dovesi, V. R. Saunders, C. Roetti, R. Orlando, C. M. Zicovich-Wilson, F. Pascale, B. Civalleri, K. Doll, N. M. Harrison, I. J. Bush, P. D'Arco, M. Llunell, M. Causà and Y. Noël, CRYSTAL14 User's Manual, University of Torino, Torino, 2014, p.

[42] K. Andersson and B. O. Roos, Chem. Phys. Lett., 1992, 191, 507-514.

[43] C. D. Graaf, R. Broer and W. C. Nieuwpoort, Chem. Phys., 1996, 208, 35-43.

[44] G. Karlström, R. Lindh, P. A. Malmqvist, B. O. Roos, U. Ryde, V. Veryazov, P. O. Widmark, M. Cossi, B. Schimmelpfennig, P. Neogrady and L. Seijo, Comput. Mater. Sci., 2003, 28, 222-239.

[45] F. Aquilante, L. D. Vico, N. Ferré, G. Ghigo, P. Å. Malmqvist, P. Neogrády, T. B. Pedersen, M. Pitonak, M. Reiher, B. O. Roos, L. Serrano-Andrés, M. Urban, V. Veryazov and R. Lindh, J. Comp. Chem., 2010, 31, 224-247.

[46] M. Douglas and N. M. Kroll, Annals of Physics, 1974, 82, 89-155.

[47] B. A. Hess, Physycal Review A, 1986, 33, 3742-3748. 